\title{
Unique life saving emergency cardiac surgery in the Cath Lab; a case report
}

\author{
Trushar Gajjar • Gaurang Shah • Neelam Desai
}

Received: 10 December 2010/Revised: 26 January 2011 / Accepted: 9 February 2011 /Published online: 8 April 2011

(C) Indian Association of Cardiovascular-Thoracic Surgeons 2011

\begin{abstract}
A 31 year old female with severe rheumatic mitral stenosis underwent percutaneous balloon valvotomy, she developed cardiac tamponade due to perforation of the right atrial wall by a guide wire. We describe this case as an emergency lifesaving procedure and as one of the indications for right thoracotomy approach for closed mitral valvotomy performed in the cardiac cath lab.
\end{abstract}

Keywords Thoracotomy $\cdot$ Rheumatic $\cdot$ Stenosis

\section{Case report}

A 31 year old female admitted to our institution with history of shortness of breath New York Heart Association class II, palpitation and history of rheumatic fever in childhood. Clinical examination revealed; Heart rate $72 / \mathrm{min}$, regular; Blood pressure 90/70 mm Hg. Loud first heart sound, loud second heart sound in pulmonary area, opening snap and mid diastolic murmur at apex. Electrocardiogram showed Sinus rhythm with left atrial enlargement. Chest X-ray

\author{
T. Gajjar $\cdot$ G. Shah $\cdot$ N. Desai \\ Sri Sathya Sai Institute of Higher Medical Sciences, \\ Prasanthigram-515134, District Anantapur, \\ Andhra Pradesh, India \\ G. Shah \\ e-mail: gaurang101@hotmail.com \\ N. Desai \\ e-mail: neelsai@gmail.com \\ T. Gajjar $(\bowtie)$ \\ Consultant - CTVS Department, \\ Sri Sathya Sai Institute of Higher Medical Sciences, \\ Prasanthigram-515134, District Anantapur, \\ Andhra Pradesh, India \\ e-mail: trushargajjar@gmail.com
}

revealed: Left atrial enlargement with straightening of left heart border. Two dimensional Echocardiogram showed, Pliable mitral valve, Mitral Valve Area 0.9 square centimeter, gradient across the mitral valve $28 / 18 \mathrm{~mm}$ $\mathrm{Hg}$, mild subvalvular fusion no clots in left atrium or appendage, trivial mitral and tricuspid regurgitation, no aortic and tricuspid stenosis or aortic regurgitation, good ventricular function, and right ventricular systolic pressure $45 \mathrm{mmHg}$.

As the valve was pliable percutaneous balloon mitral commissurotomy was planned and she was taken to the Cath lab for the procedure. Under local anaesthesia right femoral artery and vein cannulated. Repeated unsuccessful attempts were made to puncture the septum because it was thick walled and, the procedure was abandoned. At this time, the patient developed hypotension and the epicardial echocardiogram showed cardiac tamponade. A pigtail catheter was put and dark red blood was aspirated. The blood pressure improved transiently but again started falling. There was continuous flow of the dark red blood from the pericardial cavity. Patient was anaesthetized and intubated. A central venous line was put. Inotropic supports were started. Heparin was given and an urgent call for help was sent to the cardiac surgical team. The operation theaters were busy at that time and it would take at least 2 hours for them to make operation theater available. It was decided to perform the surgery in the cath lab. The patient was turned to left lateral position. The right lateral thoracotomy was done based on the surgical assumption at that point for the cause of bleeding which being dark blood was suggestive of inferior vena cavae-right atrial junction or right atrial tear. The right lung was retracted and the pericardium was opened vertically anterior to the right phrenic nerve. Large amount of clots were evacuated and the tamponade was relieved. The haemodynamics im- 
proved immediately and blood pressure came up to 50$60 \mathrm{mmHg}$, but still the pericardial cavity was welling up with dark red blood. Stay sutures were placed and suspected area of perforation (Inferior vena cavae-Right atrial junction) was explored but there was no tear visible at that site. The pericardium was opened further up and the site of perforation was visualized on the right atrium just beneath the right atrial appendage. The perforation was closed with polypropylene suture. Blood and fluids were given to fill up the heart. The mitral valve was severely stenotic and had not been dilated by the percutaneous balloon mitral commissurotomy procedure. Rapid infusion of large amount of the fluid with a stenotic mitral valve threatened to lead to acute pulmonary oedema.

The other option of attempting a balloon dilatation with open chest was difficult because of the change in position of the patient. With the experience of more than 2000 closed mitral valvotomies including redo in this hospital from the left side of the chest, it was decided to attempt a finger dilatation of the valve from the mildly enlarged left atrial body which was projecting out on the right side. A double purse string suture with 4-0 polypropylene was taken on the Left Atrial body (LA) (Fig. 1) and a stab was put and dilated with artery forcep, the index finger was introduced into the left atrium (Fig. 2) and the stenotic mitral valve was opened by finger fracture technique. The dilatation was assessed with finger which was found to be adequate for rapid infusion of the blood. Finger was removed and the purse string sutures were tied (Fig. 3). Haemostasis was confirmed. The pericardium and chest were closed after putting the drains. On table epicardial echocardiography showed mitral valve area 1.7 square centimeter, no mitral

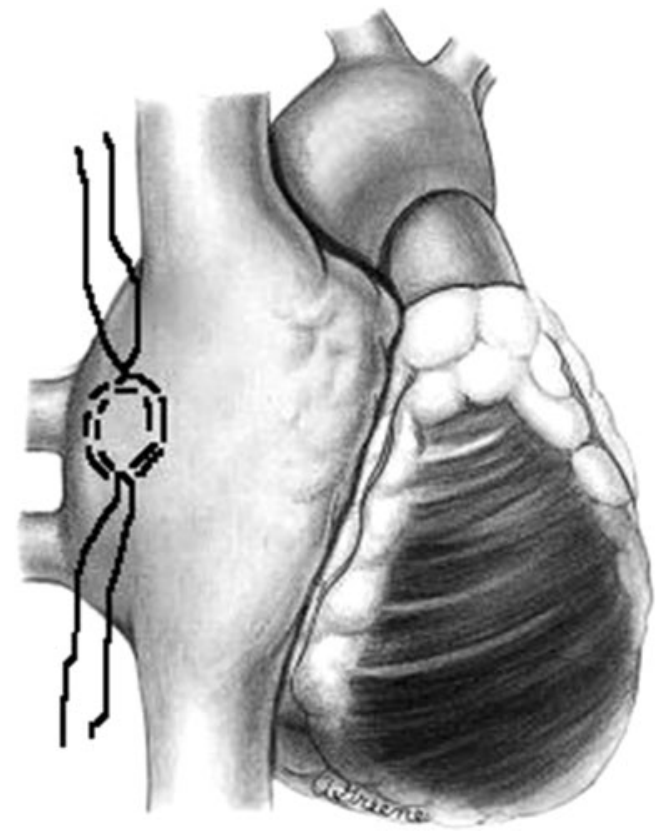

Fig. 1 Double purse string sutures over dilated Left atrium

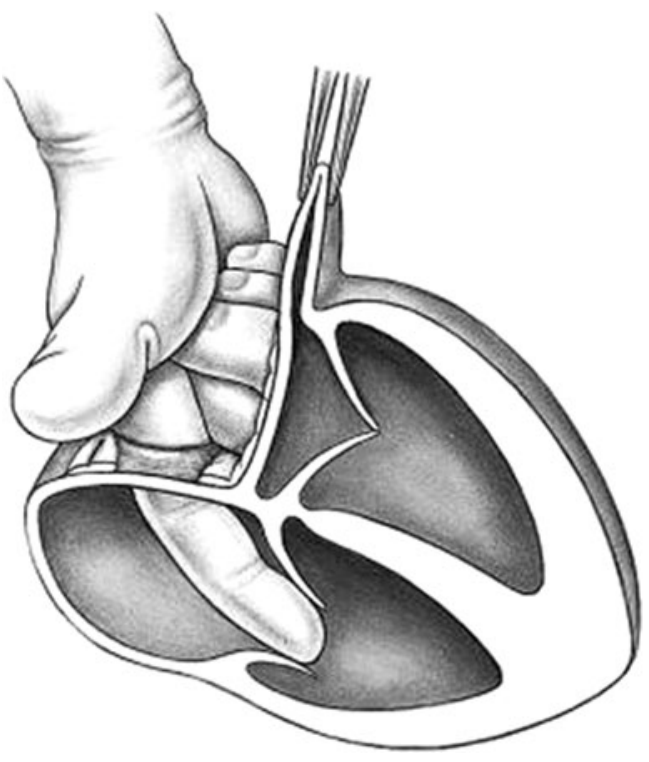

Fig. 2 Finger dilatation of Mitral valve

regurgitation, good ventricular function, collapsed left and right ventricles. The blood pressure improved after infusion of the blood. Patient was shifted to intensive care unit and was put on ventilator with inotropes. She was extubated the next day. Inotropes were gradually weaned off and was shifted to the ward on 10th post-operative day. Patient was discharged on 19th post-operative day. The echocardiogram before the discharge showed; mitral valve area of 1.7 square centimeter, gradient across mitral valve $10 / 8 \mathrm{~mm} \mathrm{Hg}$, right ventricular systolic pressure $32 \mathrm{~mm} \mathrm{Hg}$, good biventricular function, and no pericardial effusion.

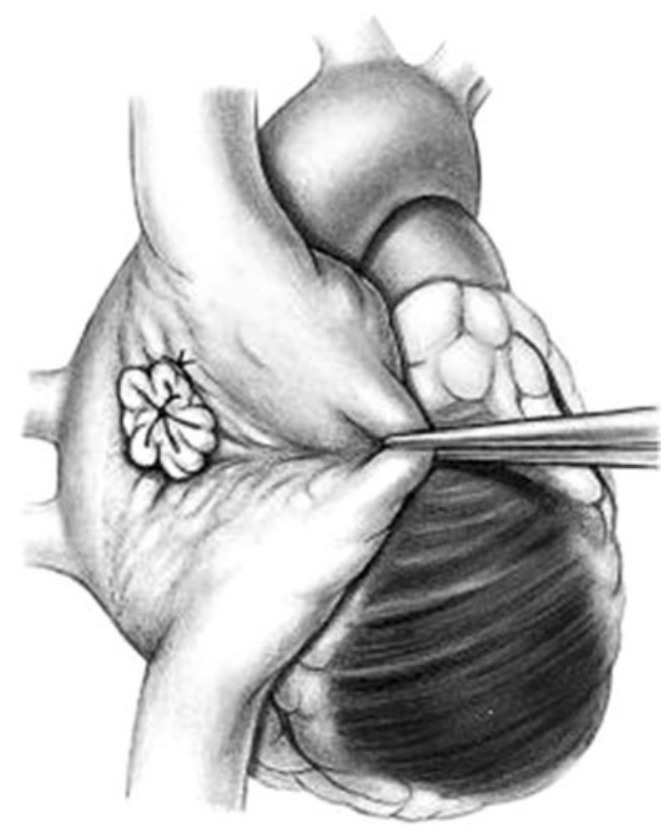

Fig. 3 Tied purse string sutures 


\section{Comments}

For many years prior to the routine use of the heart lung machine, Closed Mitral Valvotomy/Closed Mitral Commissurotomy (CMV/CMC) was the mainstay for relief of severe rheumatic mitral stenosis. CMV was 1st described by Souttar in 1925 [1]. A left thoracotomy is the most common and conventional access modality. Use of right thoracotomy is not a new concept, Neptune and Bailey described this approach in 1954 [2]. De Dang Hanh and A Thomas Pezzella from Vietnam reported 300 cases by right thoracotomy approach for redo CMV.

Indications for right thoracotomy approach are 1. Mitral stenosis with tricuspid stenosis, 2. Mitral stenosis with associated pulmonary pathology, 3. Mitral stenosis with atrial septal defect or atrioseptopexy and 4. in Redo CMV [2].

Advantages of right thoracotomy approach are, 1. no adhesions, 2. possible to avoid parenchymal tear and air leak, 3. no compromise in the pulmonary vein openings in LA, 4. less chance of embolisation of the clot, 5. finger dilatation is sufficient in younger patients, 6 . the posterior leaflet is more adherent than anterior leaflet and by this approach pulp of the finger is against the posterior leaflet which facilitates in the finger fracture technique, and 7. associated lesions can be tackled [2].

Disadvantages of right thoracotomy approach are, 1. if mitral valve is deeper access is difficult, 2. only finger dilatation is possible, 3 . difficult to pass Tubb's dilator, 4. bleeding from the finger insertion site, and 5. air embolism [2].

We would like to add one more indication for this approach as, a traumatic cardiac rupture in cath lab when the operation theatre is not available. It would have been unfortunate if the situation could not have been salvaged due to the lack of availability of operating room. Such a situation could arise in any hospital. Since these emergencies occur infrequently (less than 1\%), keeping an operation theater free for them would mean that there would be a significant loss of the operation theater time and manpower. Use of Right thoracotomy over median sternotomy is preferable because, 1. Quicker entry to the site. 2. Excellent exposure of the site of perforation 3 . Very easy to perform the finger fracture opening of the valve. 4. Future mitral valve replacement through median sternotomy will be safe and without the complications of repeat sternotomy. Hence by shifting only 3 pieces of equipment from the operating room, the cath. lab can be converted into a temporary operating room to perform this unconventional life saving surgery!

\section{References}

1. Souttar HS. The surgical treatment of Mitral stenosis. Br Med J. 1925;2:603-6.

2. De Hanh D, Pezzella AT. Closed mitral commissurotomy utilizing right thoracotomy approach. Asian Cardiovasc Thorac Ann. 2000;8:192-4. 